\title{
A Nonlinear Theory for El Niño Bursting
}

\author{
Axel Timmermann \\ Institute for Marine Research, Kiel, Germany \\ FEI-FEI JIN \\ Department of Meteorology, School of Ocean and Earth Science and Technology, Honolulu, Hawaii \\ JAN ABSHAgen \\ Institute for Marine Research, Kiel, Germany
}

(Manuscript received 11 June 2001, in final form 27 June 2002)

\section{ABSTRACT}

\begin{abstract}
A new mechanism is proposed that explains two key features of the observed El Niño-Southern Oscillation (ENSO) phenomenon-its irregularity and decadal amplitude changes. Using a low-order ENSO model, the authors show that the nonlinearities in the tropical heat budget can lead to bursting behavior characterized by decadal occurrences of strong El Niño events. La Niña events are not affected, a feature that is also seen in ENSO observations. One key result of this analysis is that decadal variability in the Tropics can be generated without invoking extratropical processes or stochastic forcing. The El Niño bursting behavior simulated by the low-order ENSO model can be understood in terms of the concept of homoclinic and heteroclinic connections. It is shown that this new model for ENSO amplitude modulations and irregularity, although difficult to prove, might explain some features of ENSO dynamics seen in more complex climate models and the observations.
\end{abstract}

\section{Introduction}

The El Niño-Southern Oscillation phenomenon (ENSO) is the strongest interannual climate mode (Neelin et al. 1998). It can be characterized by an interannual cooling (La Niña) and warming (El Niño) of the eastern equatorial Pacific. Though it originates in the tropical Pacific, it has an impact on weather and climate globally. ENSO has to be regarded as an inherently coupled atmosphere-ocean mode. Eastern equatorial Pacific temperature anomalies are accompanied by tropical wind anomalies that in turn reinforce the oceanic temperatures. Besides this positive feedback a delayed negative feedback exists, which is provided by wave propagation and advection in the tropical ocean.

In addition to the interannual climate variations associated with the ENSO phenomenon, climate variability in the Tropics can be observed also on decadal and interdecadal timescales (e.g., Graham 1994; Zhang et al. 1997; Zhang et al. 1998; Liu and Zhang 1999). There are two main hypotheses to explain this type of variability.

Corresponding author address: Dr. Axel Timmermann, Institute für Meereskunde, Theorie und Modellierung, Düsternbrooker Weg 20, Kiel, D-24105, Germany.

E-mail: atimmermann@ifm.uni-kiel.de
- Decadal variability in the Tropics is generated by extratropical air-sea interactions (e.g., Gu and Philander 1997; Kleeman et al. 1999; Pierce et al. 1999; Schneider 2000).

- Decadal tropical climate variations are generated by tropical atmosphere-ocean dynamics alone (e.g., Zebiak and Cane 1987; Münnich et al. 1991; Kirtman and Schopf 1998; Timmermann and Jin 2002).

It should furthermore be noted that different ENSO irregularity scenarios suggested in the last couple of years, such as stochastic excitation (Chang et al. 1996; Eckert and Latif 1997; Blanke et al. 1997; Moore and Kleeman 1999; Wang et al. 1999) and the nonlinear ENSO-annual cycle interaction scenario (Jin et al. 1994; Tziperman et al. 1994) can also explain a fraction of decadal climate variability observed in the Tropics.

Very often the term "decadal variability" is used in a nonspecific way. In order to be more precise it is useful to address the following questions: Is the decadal variability under consideration different, in a statistical sense, from a statistical or physical null hypothesis? Is it appropriate to deal with linear statistical techniques such as correlation analysis or multivariate stationary eigenvector-based techniques to identify decadal signals? Is the phenomenon under consideration expressed in terms of decadal changes in the mean state and/or 
decadal changes of the variability? Are these two statistical quantities interrelated?

In our study we focus on the question: What drives decadal changes of ENSO variability? Despite the fact that there is no pronounced decadal peak in the spectrum of tropical sea surface temperature anomalies (SSTAs) there is ample observational evidence ( $\mathrm{Gu}$ and Philander 1995; Torrence and Webster 1999) that the amplitude of ENSO undergoes changes on timescales of 10-20 years. ${ }^{1}$ Due to the shortness of the observational record it is difficult to distinguish firmly whether the observed ENSO amplitude modulation is the result of stationary autoregressive statistics or whether it is a manifestation of deterministic processes shaping long-term ENSO dynamics. Further support for the deterministic hypotheses comes from coupled general circulation model simulations (CGCM; see Timmermann et al. 2001). Timmermann et al. (2001) show that the ENSO dynamics simulated by the CGCM ECHAM4/OPYC can be reduced to a nonlinear four-dimensional ordinary differential equation system, that exhibits similar ENSO amplitude modulations as the full CGCM. It has been shown (Timmermann 2002) that amplitude modulations as simulated by the empirically derived low-order ENSO model originate from a homoclinic bifurcation and are not related to stochastic excitation. Whether this holds also for the observations is unknown.

We will derive a low-order nonlinear model of the tropical Pacific that explains both the existence of decadal ENSO amplitude modulations as well as decadal changes in the tropical mean state. We will also show that these statistical quantities are tightly related. Furthermore, our analysis suggests a new mechanism for ENSO irregularity. If our low-order model results were portable to reality, this would indicate that linear multivariate stationary eigenvector-based techniques might not be the appropriate statistical tools to investigate decadal variability in the Tropics.

Our paper is organized as follows. Section 2 describes the dynamical behavior of a low-order ENSO model. It is shown that ENSO amplitude modulations and ENSO chaos can be interpreted in terms of dynamical systems concepts discussed in more detail in the appendix. We will derive typical criteria that characterize the emergence of ENSO amplitude modulations. Furthermore, the role of noise and the annual cycle is discussed. Our paper concludes with a discussion and summary of our main results (section 3). In the appendix we give a brief introduction into the theory of homoclinic and heteroclinic orbits.

\footnotetext{
${ }^{1}$ Note, decadal changes in the amplitude of ENSO measure variations in the energy contained in the interannual ENSO band and should not be confused with decadal changes in the mean state of the tropical eastern Pacific.
}

\section{Nonlinear dynamics of ENSO}

\section{a. A low-order ENSO model}

In order to explore the nature of ENSO amplitude vacillations we use a low-order ENSO model (Jin 1998; Sun 2000). This model can be derived from a simplified version of the Zebiak and Cane (1987) ENSO model using a two-strip and a two-box approximation (Jin 1997). The upper ocean is a box model version of a shallow-water model for the equatorial ocean combined with a mixed layer of fixed depth (Cane 1979). According to Sun (2000), appropriate boxes are the western equatorial region extending from $135^{\circ} \mathrm{E}$ to $155^{\circ} \mathrm{W}$ and the eastern surface box extending from $155^{\circ}$ to $85^{\circ} \mathrm{W}$. The atmosphere is approximated in terms of a linear relationship between surface winds and the SST gradient. A thermal relaxation towards a radiative-convective equilibrium temperature $T_{r}$ is assumed.

The heat budget of this model can then be expressed as follows:

$$
\begin{aligned}
& \frac{d T_{1}}{d t}=-\alpha\left(T_{1}-T_{r}\right)-\frac{u\left(T_{2}-T_{1}\right)}{(L / 2)} \\
& \frac{d T_{2}}{d t}=-\alpha\left(T_{2}-T_{r}\right)-\frac{w\left(T_{2}-T_{\mathrm{sub}}\right)}{H_{m}},
\end{aligned}
$$

where $T_{1}$ and $T_{2}$ represent the eastern and western equatorial temperature, respectively; $1 / \alpha$ measures a typical thermal damping timescale; the term $T_{\text {sub }}$ denotes the temperature being upwelled into the mixed layer; $w$ is the upwelling velocity, whereas $u$ represents the zonal advection velocity, which is assumed to be proportional to the wind stress; ${ }^{2}$ and $H_{m}$ and $L$ denote the depth of the mixed layer and the basin width, respectively. It is well known that equatorial upwelling is largely due to Ekman flow divergence. Its dominating meridional component is proportional to zonal wind stress $\tau$. Thus, we obtain

$$
\begin{aligned}
u /(L / 2) & =\epsilon \beta \tau \\
w / H_{m} & =-\zeta \beta \tau,
\end{aligned}
$$

where $\epsilon$ and $\zeta$ measure the strength of the zonal and vertical advection, respectively, and will be used as bifurcation parameters. Neglecting the role of the Hadley circulation and other external sources for wind stress anomalies, the wind stress related to the Walker circulation can be expressed as

$$
\tau=\mu\left(T_{1}-T_{2}\right)\left[\gamma \cos \left(\omega_{a} t\right)+\sigma \xi_{t}-1\right] / \beta,
$$

where $\sigma \xi_{t}$ represents Gaussian white noise of variance $\sigma^{2}$ and $\gamma$ is the strength of the annual cycle of frequency $\omega_{a}$. A typical value for $\gamma$ is 0.2 . Unless otherwise stated,

\footnotetext{
${ }^{2}$ The inclusion of the advective feedback in terms of $u \overline{\nabla T}$ is discussed in Jin and An (1999) and An et al. (1999). Here we also take into account temperature tendency terms such as $u \nabla T^{\prime}$.
} 
the experiments described below neglect the annual cycle and noise (i.e., $\gamma=\sigma=0$ ). The subsurface temperature $T_{\text {sub }}$ depends strongly on the thermocline depth. Following Jin (1996) it can be parametrized as

$$
T_{\text {sub }}=T_{r}-\frac{T_{r}-T_{r 0}}{2}\left[1-\frac{\tanh \left(H+h_{2}-z_{0}\right)}{h^{*}}\right] .
$$

Here $h_{2}$ is the depth departure of the eastern equatorial thermocline from its reference depth $H, z_{0}$ is the depth at which $w$ takes its characteristic value, and $h^{*}$ measures the sharpness of the thermocline.

The east-west contrast of thermocline depth is determined by the Sverdrup balance between the pressure gradient and equatorial wind stress. We assume an instantanous adjustment (Cane and Sarachik 1981) of the thermocline gradient to wind stress changes. This relationship accounts for the short traveling time of Kelvin waves in the equatorial Pacific as compared to the dynamical adjustment time of the western equatorial $\mathrm{Pa}-$ cific thermocline. Changes of the western equatorial thermocline depth $h_{1}$ are governed by the zonally integrated Sverdrup meridional mass transport resulting from wind-forced Rossby waves. This process is characterized by the dynamical adjustment timescale $1 / r$. The dynamical equations for the thermocline depth anomalies in the eastern and western equatorial Pacific read

$$
\begin{aligned}
h_{2} & =h_{1}+b L \tau, \\
\frac{d h_{1}}{d t} & =r\left(-h_{1}-\frac{b L \tau}{2}\right),
\end{aligned}
$$

respectively, and $b$ captures the efficiency of wind stress $\tau$ in driving thermocline tilt. The wind stress $\tau=-\mu\left(T_{1}\right.$ $\left.-T_{2}\right) / \beta$ applied to the thermocline forcing does not contain an annual cycle or a noise component. The reason is that our thermocline equations are filtered equations (wave propagation processes are already filtered out). In order to account properly for the effect of noise and seasonal wind forcing on thermocline dynamics, one would have to use a model that resolves the waves. Hence, in our simple model noise and seasonal forcing are only included in the temperature equations (1) and (2). The parameter values used in our study are $T_{r 0}=$ $16^{\circ} \mathrm{C}, T_{r}=29.5^{\circ} \mathrm{C}, \alpha=1 / 180 \mathrm{day}^{-1}, r=1 / 400 \mathrm{day}^{-1}$, $H_{m}=50 \mathrm{~m}, H=100 \mathrm{~m}, z_{0}=75 \mathrm{~m}, h^{*}=62 \mathrm{~m}, \mu=$ $0.0026 \mathrm{~K}^{-1} \mathrm{day}^{-1}, \mu b L / \beta=22 \mathrm{~m} \mathrm{~K}^{-1}, \zeta=1.3$, and $L=15 \times 10^{6} \mathrm{~m}$.

The low-order model is integrated forward in time using a standard Runge-Kutta method of fourth order. In the following experiments different parameter values for $\epsilon$ are chosen, such as to explore the role of the nonlinear zonal advection term in generating chaotic behavior. A "realistic" order of magnitude for $\epsilon=$ $-2 u H_{m} / L / w \zeta$ has been estimated from a CGCM simulation (Timmermann et al. 1999). Here $\epsilon$ attains typical values between 0.05 to 0.12 with a strong latitudinal dependence; $\epsilon$ was also estimated from the Simple Ocean Data Assimilation (SODA) product (Carton et al. 2000). For the period from 1980 to 1999 and assuming a mixed layer depth of $30 \mathrm{~m}, \epsilon$ attains values between 0.024 (in eastern equatorial Pacific between $1^{\circ} \mathrm{S}-1^{\circ} \mathrm{N}$ ) and about 0.24 between $2^{\circ} \mathrm{S}-2^{\circ} \mathrm{N}$ (S.-I. An 2001, personal communication).

Figure 1 shows time series and phase space plots of the low-order ENSO model, corresponding to different values of $\epsilon$.

\section{b. Simulated features of low-order ENSO model}

For small values of $\epsilon \sim 0.098$ the system exhibits a regular low-amplitude biannual (period of 25 months) oscillation. The phase space trajectory can be interpreted in terms of the ocean recharge-oscillator paradigm (Jin 1997). Increase of $\epsilon$ to values of about 0.1 leads to qualitatively new dynamical behavior. One observes strong amplitude modulations of ENSO that are associated with a bursting of extreme El Niño events occuring on decadal and interdecadal timescales. This bursting is associated with a large positive skewness of the distribution of eastern equatorial Pacific SST anomalies. The phase space plot reveals that the large temperatures of this amplitude vacillation are associated with a flat thermocline within the tropical west Pacific. Within this regime of amplitude modulated behavior both periodic and chaotic windows exist. Large values of $\epsilon$, around 0.18 , generate a large-amplitude ENSO oscillation with a period of 3-4 years.

It should be noted here that the ratio of the extreme El Niño events to the small events is unrealistically high. This might be an artifact of our simplified model. As can be seen in Timmermann et al. (2001), a nonlinear four-dimensional empirical ENSO model produces more realistic ENSO amplitude modulations. Still, we believe that our simplified model is suited to understand the role of nonlinearities in generating El Niño bursting. The details of the dynamical changes induced by changing the advective nonlinearities can be further explored by computing power spectra (Fig. 2) for the simulations shown in Fig. 1. The power spectra are computed on the basis of 1000-yr-long time series.

The dominant frequency $\left(f \sim 0.04\right.$ month $\left.^{-1}\right)$, clearly visible in the upper panels of Fig. 2 (note the different scales on the $y$ axes), corresponds to the imaginary part of the leading eigenvalues of the ODE-system, linearized around a stationary climate state characterized by an eastern equatorial cold tongue and a western equatorial warm pool. ENSO emerges due to an oscillatory instability of this stationary climate state. Nonlinear terms in the underlying dynamical equations alter the linear dynamics as can be seen in Fig. 2. For values of $\epsilon \sim 0.1$ the dominant biannual linear frequency is strongly suppressed, and enhanced variability can be observed on interannual to decadal timescales. This broadband spectral regime characterizes the chaotic 

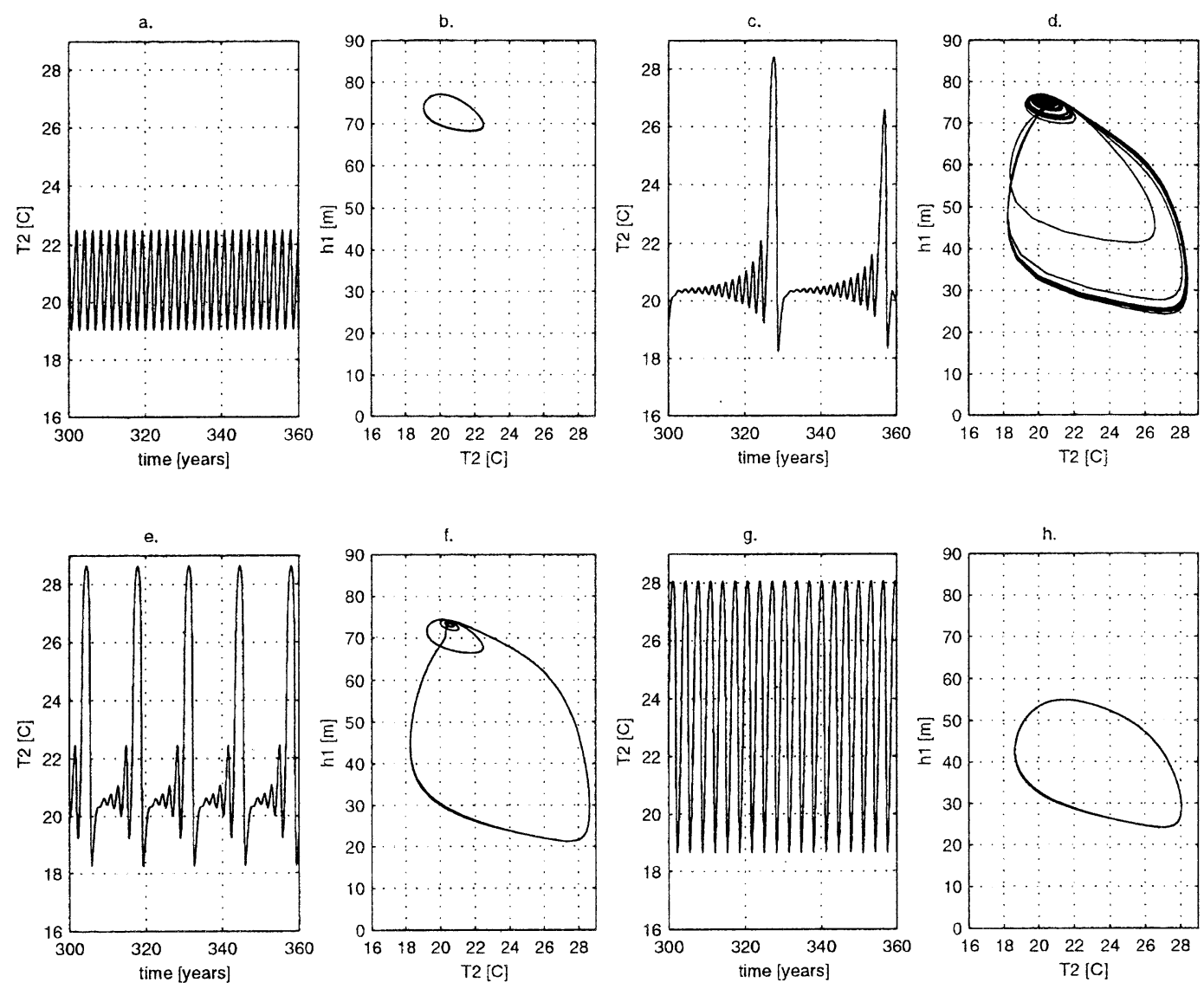

FIG. 1. (a),(c),(e),(g) Simulated time series of the eastern equatorial temperature $T_{2}\left({ }^{\circ} \mathrm{C}\right)$ and $(\mathrm{b}),(\mathrm{d}),(\mathrm{f}),(\mathrm{h})$ phase space plots [eastern equatorial temperature $T_{2}\left({ }^{\circ} \mathrm{C}\right)$ and western thermocline depth $\left.h_{1}(\mathrm{~m})\right]$ corresponding to different values of the zonal advection efficiency $\epsilon$ of (a),(b) 0.098; (c),(d) 0.1; (e),(f) 0.11; (g),(h) 0.18 .

ENSO amplitude modulation shown in Fig. 1. The spectral characteristics are very similar for the periodic oscillation at $\epsilon=0.11$. For larger $\epsilon$ values of around 0.18 the low-order model simulates a periodic ENSO cycle with a spectral peak at 3-4 years. Hence, an increase of the zonal advection can lead to a change from a lowvariance biannual to a high variance 4-yr ENSO regime. Intermediate strengths of the zonal advection parameter lead to chaotic and amplitude-modulated behavior. This clearly shows that, at least within this simplified model, decadal variability can be generated within the Tropics just as a result of advective nonlinearities.

Recently Jin and An (1999) showed that increases of the zonal equatorial currents can be an important factor in shaping ENSO variability. Their results are confirmed by our analysis.

Based on these sensitivity experiments the physical mechanism for the simulated ENSO amplitude modulations can be summarized as follows. The tropical climate mean state as represented by a fixed point of the nonlinear ENSO model becomes unstable, giving rise to ENSO-like oscillations. The eigenvalues corresponding to this interannual ENSO mode characterize a growing oscillation in one direction and a stable nonoscil- latory mode in a transversal phase-space direction. This damped mode is largely associated with the dynamics of the western equtorial Pacific [see Eq. (8)]. Hence, the climate mean state represents a saddle node of the system. For a large El Niño event the zonal temperature gradient and, hence, also the wind stress $\tau$ are close to zero. This is also associated with a minimum zonal and vertical temperature advection. In the aftermath of a strong El Niño event the warm pool depth deepens as a result of the thermocline equation (8), and the system is reset back to its climatological mean state. Subsequently, the ENSO mode grows again in its amplitude until another extreme El Niño event is reached.

\section{c. Bifurcation analysis of low-order ENSO model}

In order to study the bifurcation structure of our loworder model more in detail we perform a bifurcation analysis in which $\epsilon$ and $\zeta$ are used as bifurcation parameters. The bifurcation analysis is done in two steps: 1) continuation of equilibrium points, detection of Hopf bifurcations, and computing the stability of the fixed point solutions and 2) continuation of the periodic solutions, which emerge from the Hopf bifurcations, by 

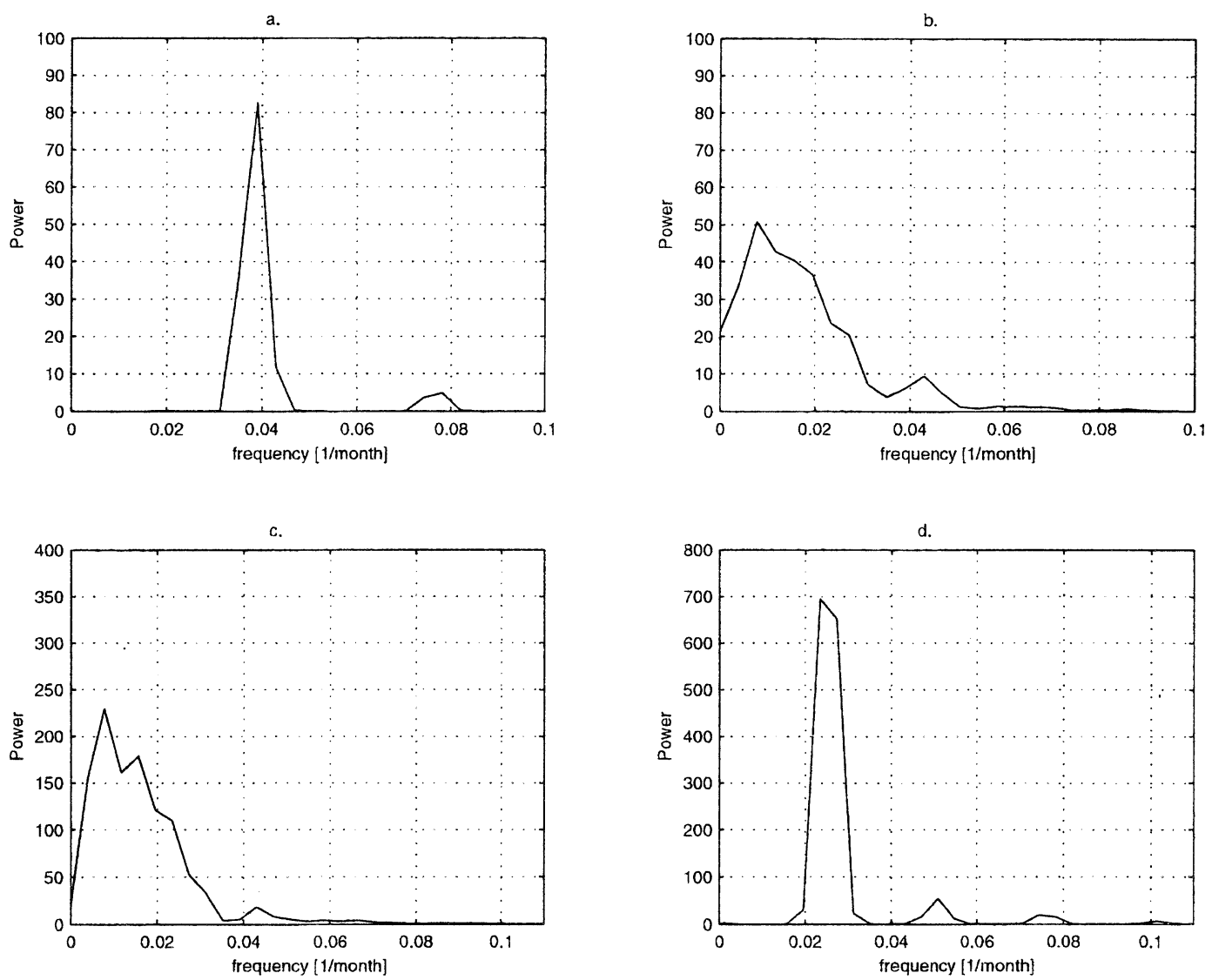

FIG. 2. Power spectra of eastern equatorial temperature anomalies, corresponding to the time series in Fig. 1. Frequency unit is one month $^{-1}$. The figure titles correspond to the parameter values of $\epsilon$ chosen for the simulations [(a) 0.098, (b) 0.1, (c) 0.11 , (d) 0.18].

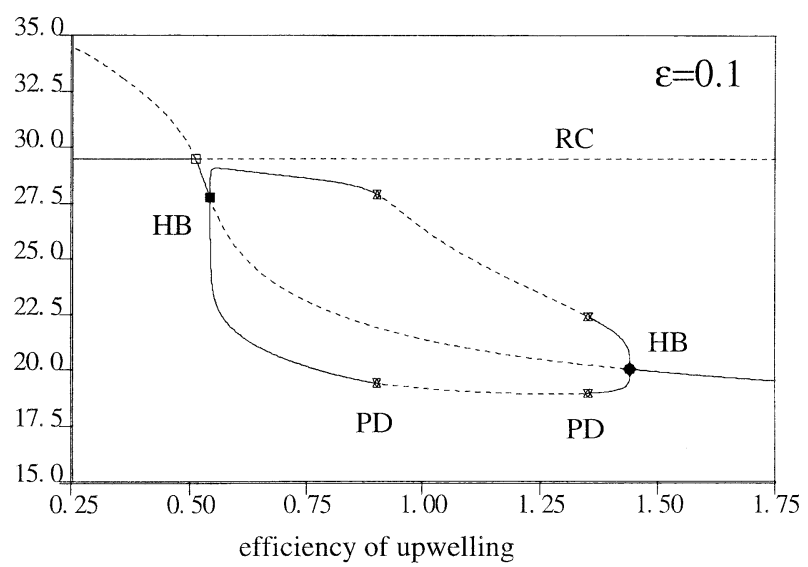

FIG. 3. Bifurcation diagram of $T_{2}$ as a function of the upwelling efficiency $\zeta$ displaying stationary and oscillatory solutions of Eqs. (1)-(2) as well as their stability. The amplitude of ENSO is represented by the curves above (maximum value of $T_{2}$ ) and below (minimum value of $T_{2}$ ) the oscillatory unstable stationary state. A dashed amplitude curve symbolizes an unstable periodic orbit. Square filled symbols represent Hopf bifurcation points, PD denotes a period doubling bifurcation and transcritical bifurcations (TBs) are symbolized by an unfilled square. RC denotes the radiative-convective equilibrium saddle point. Stable solutions are marked with solid lines, whereas dotted lines are used for unstable solutions. computing stability of periodic orbits and their bifurcations. The continuation of fixed points and periodic solutions is performed using the toolkit AUTO (Doedel 1981; Doedel et al. 1991). This software package computes branches of stable and unstable equilibria and locates and classifies bifurcations. In order to disentangle the underlying nonlinear dynamics associated with the amplitude modulations discussed in the previous section we will compute bifurcation diagrams of $T_{2}$ as a function of the vertical and zonal advection parameters $\zeta$ and $\epsilon$, respectively.

Figure 3 displays the stability diagram with respect to the vertical advection parameter $\zeta$. We have chosen the realistic value $\epsilon=0.1$. The results can be summarized as follows: for $\zeta<0.51$ (unrealistically small values) a stable and an unstable fixed point coexist. One solution corresponds to the radiative-convective equilibrium state characterized by a flat thermocline, the other one corresponds to an unrealistic permanent warm state. For $\zeta \sim 0.51$ a transcritical bifurcation leads to a change in the roles of the stationary states. For slightly larger $\zeta$ values of around 0.54 a Hopf bifurcation emerges, giving birth to the oscillatory self-sustained ENSO mode. In the vicinity of the Hopf bifurcation the ENSO mode has very long periods (on the order of several 


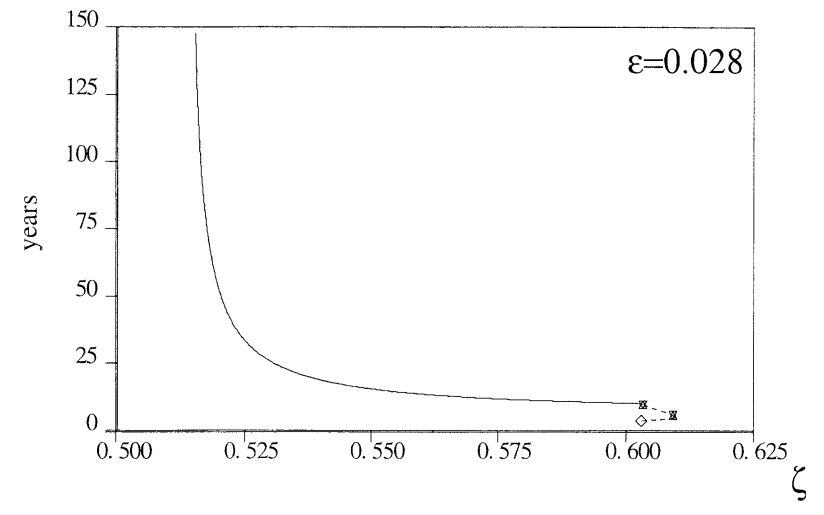

FIG. 4. Period of the ultralow-frequency mode as a function of the upwelling efficiency $\zeta$. The zonal advection strength is fixed to $\epsilon=$ 0.028. The diamonds represent period doubling bifurcations.

decades) that is a manifestation of an interaction between the limit cycle and the unstable stationary state RC. This feature becomes even more pronounced for small values of $\epsilon$ of around 0.028 , when the periodic orbit comes arbitrarily close to the unstable RC equilibrium state at $T_{2}=29.5^{\circ} \mathrm{C}$.

For $\epsilon=0.028$ and $\zeta=0.515$ a homoclinic orbit can be identified with infinite period (see Fig. 4) (see Glenndinning and Sparrow 1984). This is a codimension-1 phenomenon. Despite the fact that this corresponds to a highly unrealistic ${ }^{3}$ situation it is interesting to realize that the periodicity of ENSO is strongly modified by the interaction between the oscillatory ENSO mode and the unstable RC mean state. It should be noted here that the homoclinic orbit identified in Fig. 4 is not directly relevant for the simulated bursting behavior of ENSO in our low-order ENSO model.

A key feature that translates from the unrealistic parameter range of Fig. 4 to more realistic parameter settings is the interaction between ENSO and the unstable RC mean state. Returning now to Fig. 3 we discuss the range of $\zeta>0.54$, which corresponds to the "classical" ENSO limit cycle regime. Typical periods are on the order of several years. Increasing the value of $\zeta$ to realistic values of about 1 leads to the creation of period doubling bifurcations ${ }^{4}$ for $\zeta \sim 0.88$. If the upwelling efficiency $\zeta$ becomes unrealitically strong $(\zeta \sim 1.5)$ we observe the destruction of the oscillating ENSO regime via an inverse Hopf bifurcation. In that case the system's dynamics is trapped in an eastern equatorial cold state, in contraposition to the small- $\zeta$ case. We have seen that the flat thermocline ( $\mathrm{RC}$ equilibrium) solution plays an important role in shaping ENSO dynamics. This solution has the property to "repel" the attractor whenever the actual state of the system comes close to it. Within the limit cycle regime $(0.54<\zeta<0.88)$ the presence

\footnotetext{
${ }^{3}$ See Torrence and Webster (1999).

${ }^{4}$ Only one period doubling bifurcation is displayed here. In fact a whole cascade can be identified using other techniques.
}

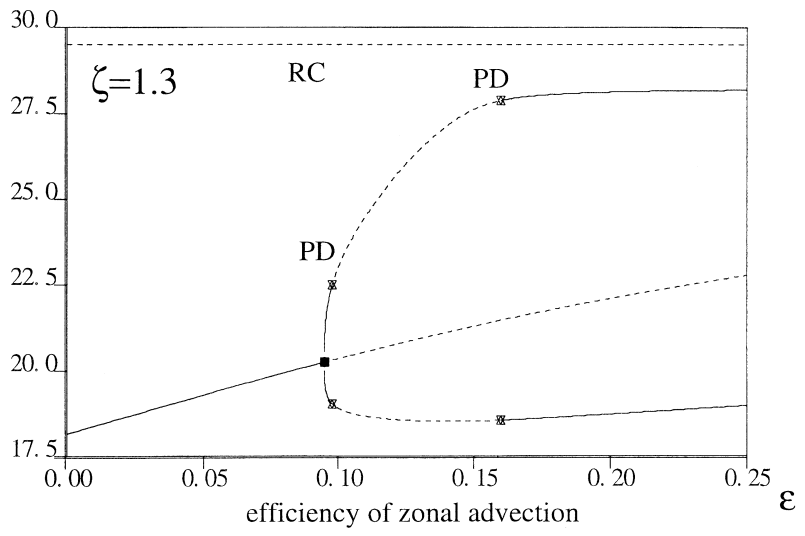

FIG. 5. Bifurcation diagram of $T_{2}$ as a function of $\epsilon$ displaying stationary and oscillatory solutions to Eqs. (1)-(2) as well as their stability. Symbols as in Fig. 3.

of an unstable equilibrium state leads to a modification of ENSO periodicity. For the unstable oscillatory regime in between the period doubling bifurcations $(0.88<\zeta$ $<1.3$ ) the existence of the unstable RC mean state has the consequence that after extreme El Niño events the phase-space trajectory is bent back into the saddle focus leading to a phase of calm ENSO activity. Summarizing the dynamics in the parameter window $0.88<\zeta<1.3$ : If the initial state is close to the saddle focus, the trajectory will spiral outwards, thereby gaining amplitude. When the system gets close to the RC equilibrium, it will be repelled from it. Eventually the system returns back into the vicinity of the saddle focus. This behavior is nicely illustrated in Fig. 1. This scenario bears many similarities to the Shil'nikov scenario (Shil'nikov 1965, 1970) for homoclinic orbits. However, our case is more complicated since the unstable RC equilibrium state is responsible for the reinjection into the saddle focus. Thus, the characteristics are more reminiscent of a heteroclinic connection. Unfortunately, there is not much literature on heteroclinic connections between saddle points and saddle foci. The time-inverse case to our situation is discussed briefly in Wiggins (1988). It is very difficult to construct analytically an explicit Shil'nikov map for our system. However, forward integrations (as shown in Fig. 1), have revealed that this special heteroclinic situation gives birth to deterministic chaos and amplitude modulations.

In section $2 b$, we have studied the sensitivity of ENSO with respect to the zonal advection parameter $\epsilon$ and found that decadal ENSO amplitude modulations can be simulated for realistic values of this parameter. In order to study this parameter dependence more in detail we have computed the bifurcation diagram of $T_{2}$ with respect to $\epsilon$. The upwelling strength $\zeta$ is fixed to 1.3. The results of this analysis are depicted in Fig. 5. We observe the unstable RC solution at $T_{2}=29.5^{\circ} \mathrm{C}$, which influences the dynamics of ENSO when an El Niño comes close to it. For small zonal advection parameters a stable equilibrium exists with complex eigenvalues 


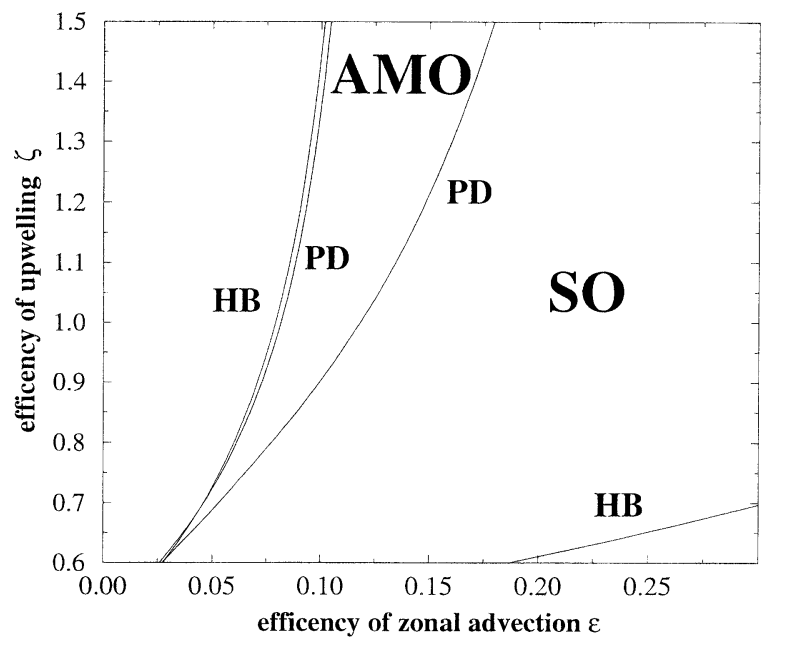

FIG. 6. Regime diagram showing ENSO limit cycle (SO) solutions and amplitude-modulated oscillatory (AMO) ENSO solutions within the $\epsilon-\zeta$ plane. HB and PD denote the Hopf bifurcation and period doubling bifurcation border lines in parameter space.

(damped oscillatory ENSO mode), which corresponds to an eastern cold tongue and a western warm pool. This mean state becomes unstable via a Hopf bifurcation at the realistic value of $\epsilon \sim 0.09$ (Jin 1998). The newly born ENSO limit cycle experiences further disturbances by the unstable RC state, giving rise to period doubling bifurcations, deterministic low-dimensional chaos, and amplitude modulated behavior (see Fig. 1). Finally, the ENSO limit cycle is destroyed by an inverse Hopf bifurcation for unrealistically large $\epsilon$ values.

In order to show that our scenario is quite robust in parameter space we compute all those situations in $\zeta-$ $\epsilon$ parameter space that are characterized by amplitude modulations. The result of our analysis, which is also performed with AUTO, is depicted in Fig. 6. The right HB curve separates the damped oscillatory ENSO mode from the self-sustained ENSO regime. The limit-cycle ENSO regime (SO) breaks up when the system crosses the right $\mathrm{PD}$ curve from right to left. In between the PD curves ENSO dynamics is characterized by amplitude-modulated behavior. Within this regime both periodic and chaotic windows exist. A small slice to the left of the left PD curve separates a damped ENSO mode, a limit cycle and the amplitude modulation regime. We observe that, in the realistic parameter window $(0.05<\epsilon<0.15,0.7<\zeta<1.3)$, about $30 \%$ of the area in parameter space is filled with amplitudemodulated situations. It is unlikely that $\epsilon$ and $\zeta$ remain the same for different climate background conditions. If the real ENSO system undergoes secular changes in which the relative importance between upwelling and zonal advection is modified (changing $\zeta$ and $\epsilon$ values), it is quite likely that the ENSO system spends some time in an amplitude-modulated oscillatory regime.

Summarizing, it can be said that the three-dimensional ENSO system has an incredibly rich dynamical behavior as can be seen from forward integrations and bifurcation analysis. In particular, the emergence of decadal ENSO amplitude modulations arises from an interaction between an ENSO limit cycle mode and the $\mathrm{RC}$ equilibrium state. This finding is completely new and has not been discussed in ENSO literature yet. It can be regarded as a new nonlinear mechanism to generate decadal climate variability within the Tropics and provides a theoretical explanation for the decadal bursting of El Niño.

\section{d. Role of annual cycle}

It has been recognized (Jin et al. 1994; Tziperman et al. 1994; Chang et al. 1996) that ENSO interacts with the annual cycle such as to give rise to irregular, chaotic behavior. Furthermore, frequency and phase locking to the annual cycle can be established by nonlinear dynamics. It is therefore crucial to study the effect of annual cycle forcing in our low-dimensional ENSO system. The numerical simulations described below are conducted for parameter values $\gamma \neq 0$. Note again, that the seasonal cycle forcing enters only the temperature equations (1) and (2).

Figure 7 displays the simulated eastern equatorial temperatures and phase-space plots for different values of the annual cycle strength $\gamma$. One observes a decrease in the return time of major El Niño events as the annual cycle strength is increased. This is a manifestion of the frequency-locking tendency to the external forcing frequency. At the same time ENSO chaos is destroyed for values $\gamma=0.1-0.2$. This effect can be understood intuitively by choosing a very large value of $\gamma$. In this case, the tropical climate system is slaved completely by the annual cycle forcing, and the forced solution is phase and frequency locked to the external periodic forcing. For weaker $\gamma$ interaction among ENSO and the annual cycle gives rise to quasi-periodic and/or chaotic dynamics.

The effect of the annual cycle forcing in our simple ENSO model is to introduce an inverse period-doubling cascade as $\gamma$ increases, which reflects different stages of nonlinear resonances. These results are consistent with the studies of Tziperman et al. (1994) and Jin et al. (1994).

Furthermore, from Fig. 7 it becomes apparent that the annual cycle has another interesting effect on the simulated ENSO statistics. It rectifies the tropical climate mean state, as well as the level of ENSO variability. This is illustrated in Fig. 8. We observe that an increased amplitude of the annual cycle goes along with an increase in the eastern equatorial Pacific mean state temperature, as well as its variability. This nonlinear effect has not been considered in the literature yet.

\section{e. Role of noise}

Another source of ENSO irregularity is stochastic weather noise. A prominent example of this effect is the 

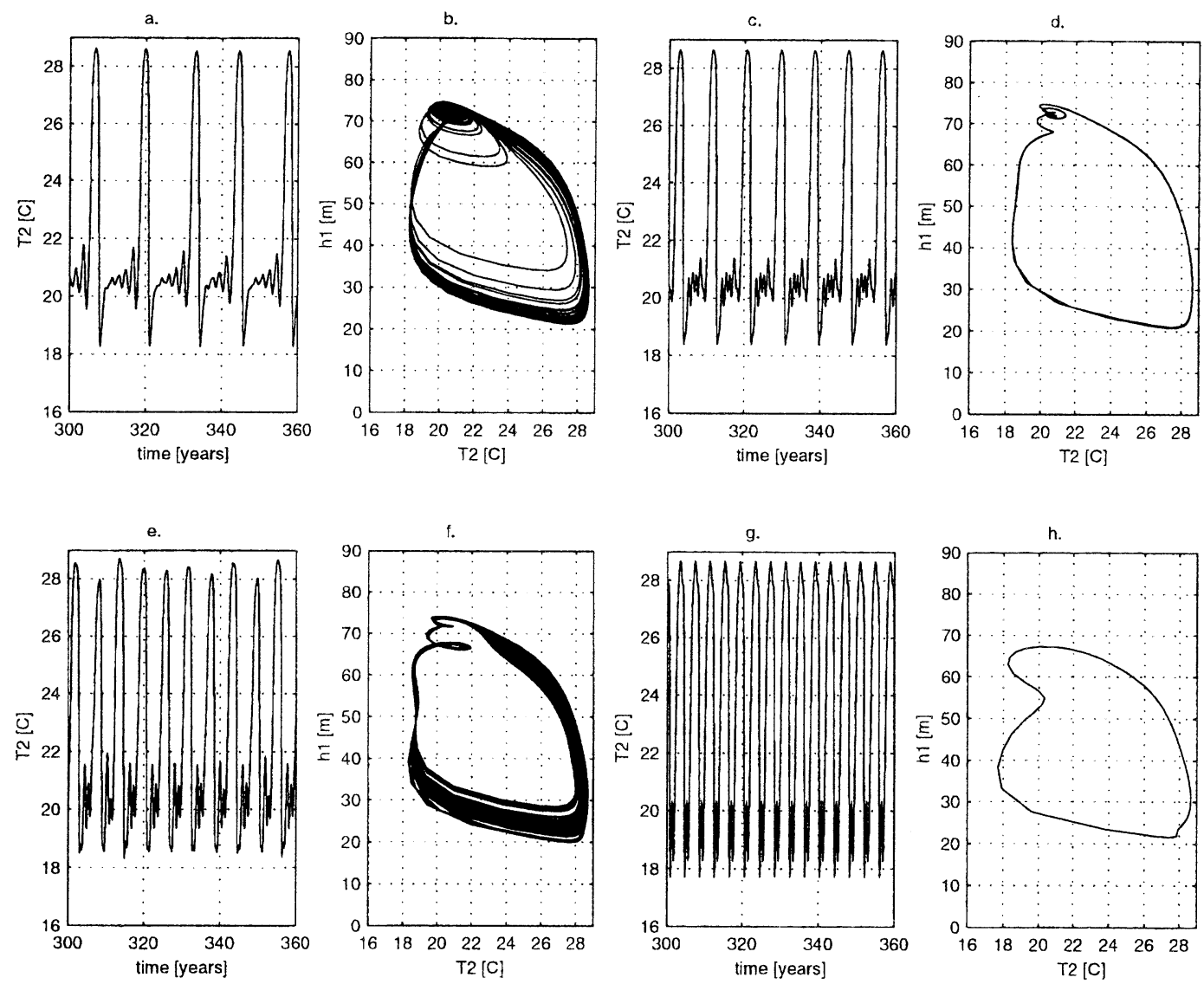

FIG. 7. (a),(c),(e),(g) Simulated time series of the eastern equatorial temperature $T_{2}$ and (b),(d),(f),(h) phase space plots (western and eastern equatorial temperature and western thermocline depth) corresponding to different values of the annual cycle strength $\gamma=0.01$ (a),(b) 0.1 ; (c),(d) 0.2 ; (e),(f) 0.5 ; (g),(h) $\zeta=1.3$ and $\epsilon=0.11$.
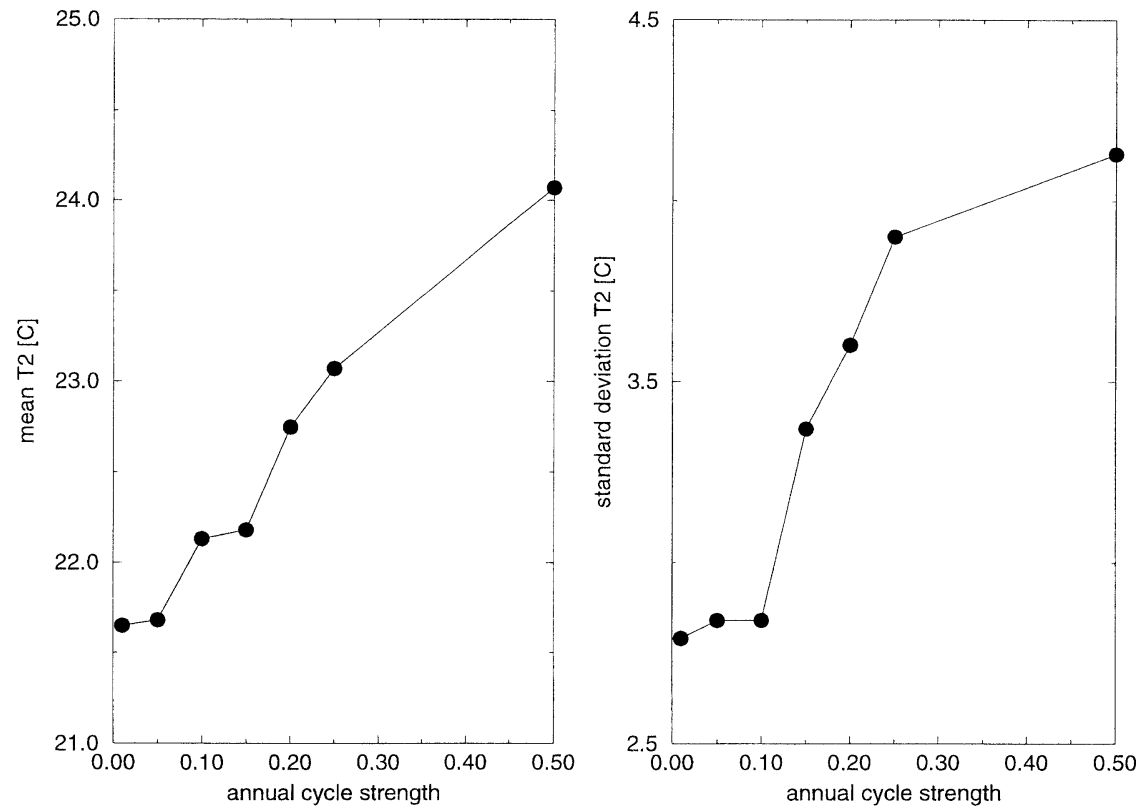

FIG. 8. Mean value and standard deviation of the simulated eastern equatorial time series shown in Fig. 7 for different values of the annual cycle strength $\gamma$. 

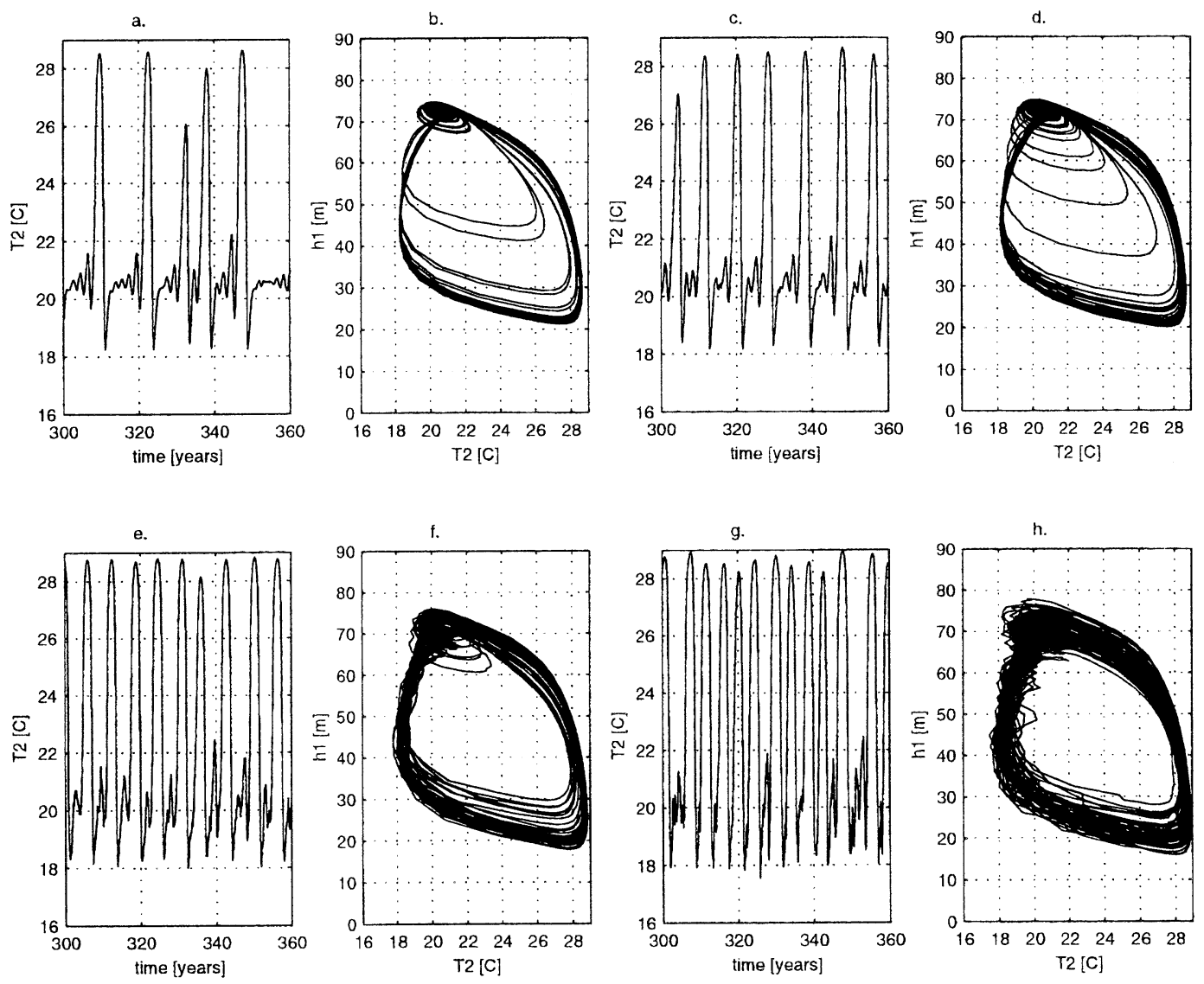

FIG. 9. (a),(c),(e),(g) Simulated time series of the eastern equatorial temperature $T_{2}\left({ }^{\circ} \mathrm{C}\right)$ and $(\mathrm{b}),(\mathrm{d}),(\mathrm{f}),(\mathrm{h})$ phase space plots [eastern equatorial temperature $T_{2}\left({ }^{\circ} \mathrm{C}\right)$ and western thermocline depth $\left.h_{1}(\mathrm{~m})\right]$ corresponding to different values of the noise strength: (a),(b) 0.1 ; (c),(d) 0.3 ; (e),(f) $1.2 ;$ (g),(h) 2.4 .

influence of short-term westerly wind bursts on the release of warm pool waters, preceeding an El Niño event. In order to study this effect we include stochastic white ${ }^{5}$ noise into our low-order ENSO model. The way this is done here is to perturb the wind stress by Gaussian white noise. It is important to note that the noise component enters the temperature equation in a multiplicative way. We will not discuss the effect of different noise colors here (Wang et al. 1999).

It can be seen from Fig. 9 that further irregularity is introduced in addition to the heteroclinic irregularity. Figure 9 illustrates that the mean bursting interval between large El Niño events is decreased by increasing the noise amplitude. However, the overall structure of the attractor remains similar to the no-noise case: large amplitude El Niño events alternate with small events. These results illustrate that the heteroclinic chaos scenario is robust against reasonable changes of the noise level, but less robust against the inclusion of an annual cycle.

\footnotetext{
${ }^{5}$ Whether this is a justifyable assumption for the tropical Pacific shall not be discussed here.
}

\section{f. Combined effect of annual cycle and noise}

The next step is to introduce both effects $(\gamma, \sigma \neq 0)$, that is, noise and seasonal cycle forcing. We choose the initial situation in which the annual cycle strength amounts to 0.5 , which corresponds to a 4-yr large amplitude ENSO cycle. At the same time the noise level is increased from $\sigma=0.1$ to 0.9 . The results of these experiments are shown in Fig. 10. One observes that the inclusion of noise revives the original homoclinic orbit structure. Furthermore, an increase in the noise level leads to farther-spaced large El Niño events as compared to the no-noise case.

Hence, parameter noise offers the possibility to bring the system trajectory back into the vicinity of the heteroclinic orbit. This illustrates that noise has a constructive effect for our nonlinear scenario rather than a destructive one. This proves that the theory suggested here is rather robust with respect to external perturbations.

Our results suggest that trajectories close to a heteroclinic orbit can be regarded as a nonlinear example of amplitude vacillations. Long timescales, which characterize the envelope of the fundamental ENSO oscillation, are introduced by the intrinsic (advective) non- 

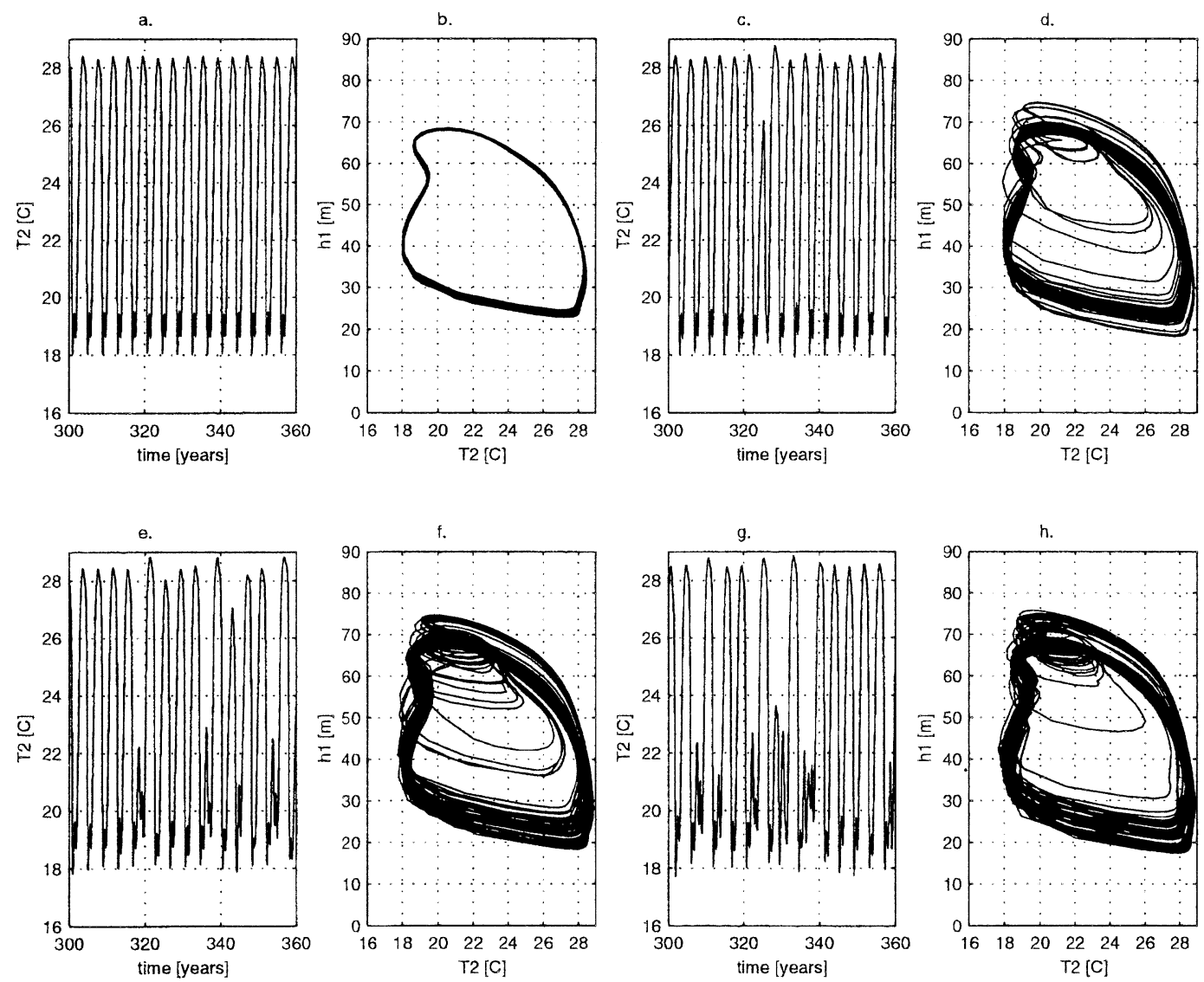

Fig. 10. (a),(c),(e),(g) Simulated time series of the eastern equatorial temperature $T_{2}\left({ }^{\circ} \mathrm{C}\right)$ and $(\mathrm{b}),(\mathrm{d}),(\mathrm{f}),(\mathrm{h})$ phase space plots [eastern equatorial temperature $T_{2}\left({ }^{\circ} \mathrm{C}\right)$ and western thermocline depth $h_{1}(\mathrm{~m})$ ] corresponding to different values of the noise strength: (a),(b) 0.1 ; (c),(d) 0.3 ; (e),(f) $0.6 ;(\mathrm{g}),(\mathrm{h}) 0.9$. The annual cycle strength is set to the realistic value of 0.2 .

linearities rather than by extratropical-tropical exchange as proposed in previous studies (e.g., Weaver 1999).

\section{El Niño bursting in complex climate models and the observations}

One crucial question to be answered here is as to whether the bursting behavior found in the low-order ENSO model can be found also in more complex climate models and also in the observations. In Timmermann et al. (2001) and Timmermann (2002) it is shown that multicentury CGCM simulations performed with the climate models ECHAM4/OPYC3 (Timmermann et al. 1999) and ECHAM4/Hamburg Ocean Primitive Equation Global Ocean-Sea Ice General Circulation model (HOPE-G; K. Rodgers 2001, personal communication) exhibit decadal ENSO amplitude modulations that are associated with a particular kind of bursting behavior. It has been shown (Timmermann et al. 2001) using nonlinear inverse modeling techniques that these amplitude modulations are due to nonlinear low-dimensional dynamics. Furthermore, Timmermann and Jin (2002) have found that the intermediate Zebiak-Cane ENSO model (Zebiak and Cane 1987) operating on an annual mean background state exhibits similar ENSO bursting behavior as the low-order model discussed here when the zonal temperature advection is increased.

Figure 11b illustrates that ENSO bursting behavior can be seen also in the observations. The Niño-3 SSTA time series is shown for the period from 1965-2001. To further highlight the similarity between the major El Niño events 1972-73, 1982-83, and 1997-98, the 198283 El Niño event is chosen as a template (thick line) for the strong El Niño events during this period. This idea is adopted from a recent paper by Douglass et al. (2002). We see that in all three cases major El Niño events are preceeded by mini El Niño events. This is more an indication rather than a proof that El Niño bursting behavior occurs also in reality. Further evidence for ENSO amplitude modulations is reported in recent papers by Gu and Philander (1995) and Torrence and Webster (1999). The observed ENSO bursting, as shown in Fig. 11b, can be compared with the dynamics of the low-order ENSO model depicted in Fig. 11a. Despite too large amplitudes in the low-order model some qualitative similarities of the observations are reproduced. Admittedly, this bursting behavior is not typical for the whole observed Niño-3 SSTA time series, 
a.

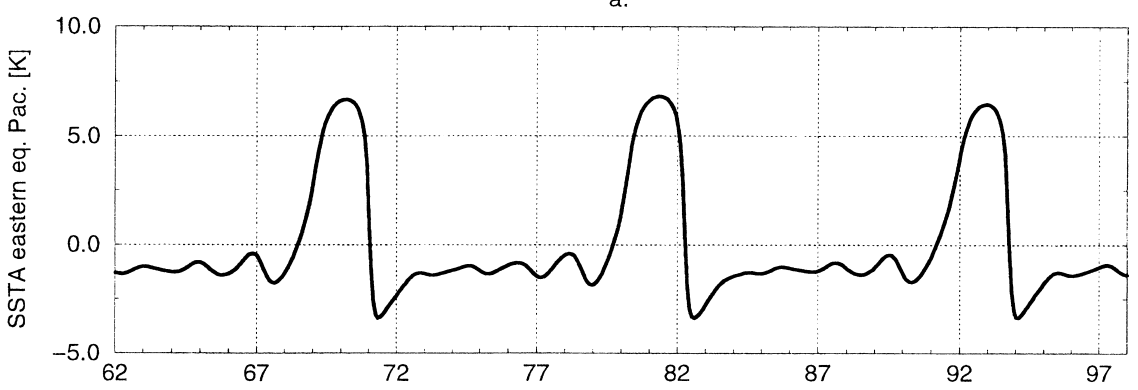

b.

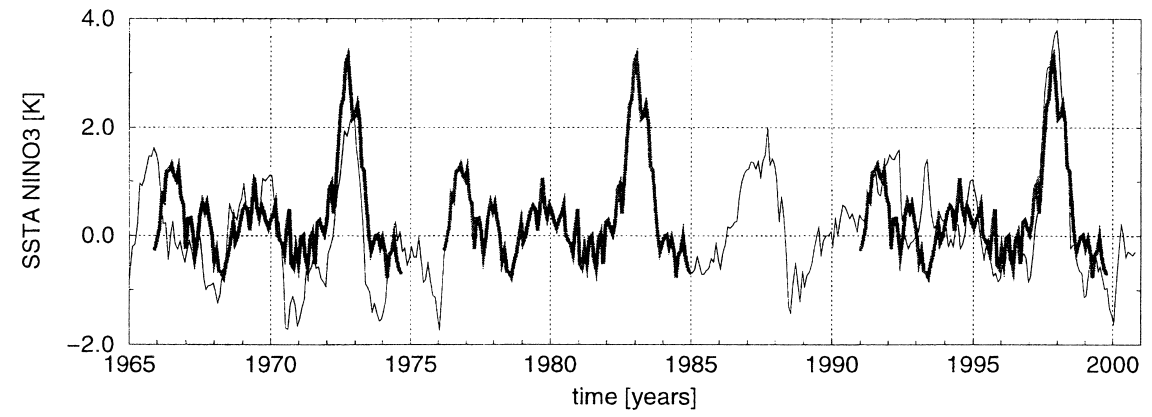

FIG. 11. (a) Simulated time series of the eastern equatorial temperature anomalies. The bifurcation parameters were set to $\zeta=1.3$ and $\epsilon=0.11$. (b) Thin: observed Niño-3 SSTA time series; thick: Niño-3 SSTA template from 1976 to 1985.
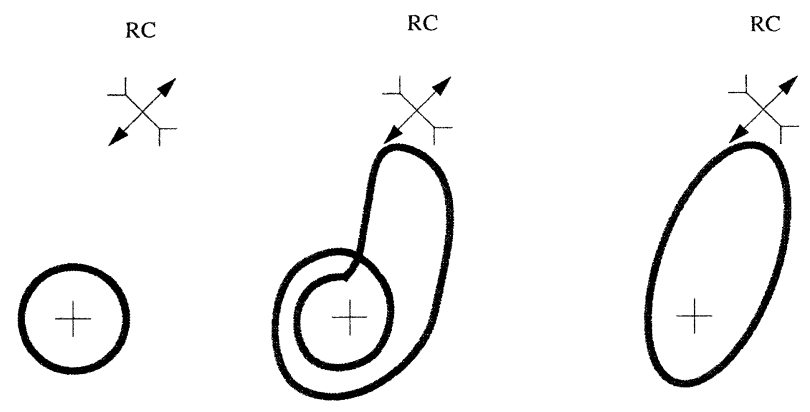

$\varepsilon$ increases

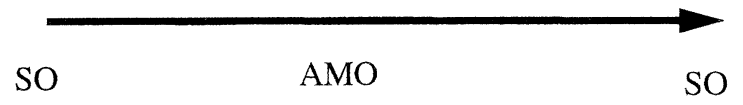

FIG. 12. Schematical phase-space representation of the amplitude modulation mechanism. Small $\epsilon$ values (left) are associated with small east to west temperature advection. The basic dynamics can be explained in terms of the recharge mechanism. Very large values of $\epsilon$ (right) lead to large ENSO variability because the strong negative advection feedback for warm pool temperatures expressed as $-\epsilon\left(T_{2}\right.$ $\left.-T_{1}\right)^{2}$ leads to a reduction of $T_{1}$, reduced trade winds, and thus, large El Niño events. During a large El Niño event, however, the zonal advection term becomes small and the recharging mechanism is initiated. (middle) intermediate values of $\epsilon$ lead to an ambivalent situation, mixing features from the low and high $\epsilon$ case, thereby generating amplitude modulations. The + indicates an oscillatory unstable fixed point, and $\mathrm{RC}$ represents a saddle point associated with the radiative-convective equilibrium state. but just for the last three decades. But it should be noted also that background conditions in the tropical Pacific have changed significantly during the last century.

\section{Discussion and summary}

It is widely accepted (e.g., Torrence and Webster 1999) that the amplitude of ENSO varies from decade to decade and that very strong El Niño conditions occur in intervals of 10-20 years. What is not understood is why. The decadal variations in ENSO amplitude might just be an expression of ENSO being partly excited stochastically or of deterministic dynamical processes interacting with ENSO. In particular, in light of recent observational studies (Torrence and Webster 1999; Gu and Philander 1995) that indicate that the amplitude of ENSO is modulated on a distinct timescale of 15-20 years, the latter possibility is quite likely and has to be investigated more in detail.

Our study revealed that a low-order ENSO model capturing the most important ENSO-relevant processes can generate amplitude modulations and El Niño bursting behavior on rather distinct decadal and interdecadal timescales, due to internal advective nonlinearities. Our scenario is based on equatorial atmosphere-ocean dynamics alone and does not invoke extratropical forcing (in agreement with Kirtman and Schopf 1998). The generation of decadal tropical variability can be understood in terms of the dynamical systems concept of heteroclinic connections. This rather general mathematical 


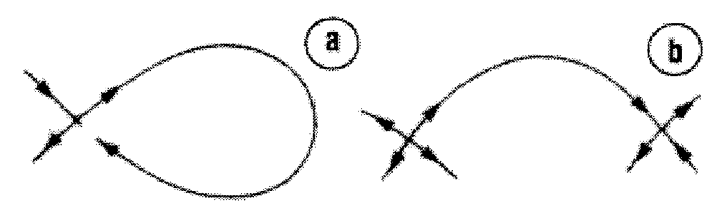

Homoclinic (a) and heteroclinic (b) orbits on the plane

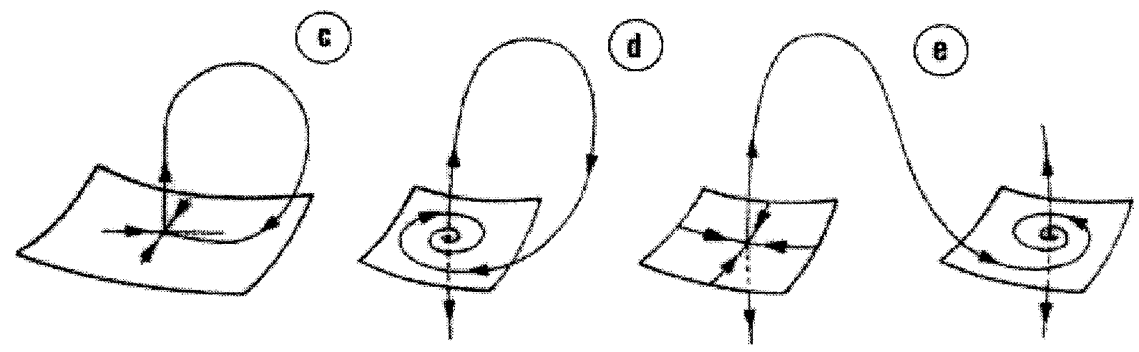

Homodinic $\left(\mathrm{c}_{8} \mathrm{~d}\right)$ and hateroclinic (e) orbits in three-dimensional space

FIG. 13. Schematic illustration of (a),(c),(d) homoclinic and (b),(e) heteroclinic orbits in two and three dimensions.

concept has implications for the simulation of the ENSO envelope period, as well as for ENSO irregularity. Our study illustrated that this nonlinear tropical scenario, which is fundamentally different from the linear extratropical hypothesis (e.g., $\mathrm{Gu}$ and Philander 1997; Schneider 2000), is robust against the combined effect of annual cycle and stochastic forcing. Admittedly, the idea of homoclinic/heteroclinic ENSO connections is less intuitive than previously suggested ideas that account for the slow tropical background changes caused by remote extratropical dynamics (e.g., Gu and Philander 1997; Schneider 2000; Weaver 1999). The fundamentally new aspect of our hypothesis is that it does not deal with a "classical" memory timescale, due to delayed ocean dynamics providing a memory on decadal timescales, but rather with a nonlinear combination of different intrinsic timescales. Furthermore, an interaction between ENSO and the radiative-convective equilibrium state occurs that is crucial for the amplitude modulated behavior and chaos. This interaction is schematized in Fig. 12.

Our theory is an alternative to previously suggested nonlinear ENSO irregularity scenarios (Jin et al. 1994; Tziperman et al. 1994). It fundamentally differs from these studies, as our mechanism can explain the generation of chaos without invoking external annual cycle forcing.

Furthermore, we found that the annual cycle has a rectifying effect on the tropical mean state. This effect might have implications for the interpretation of paleo ENSO data (Tudhope et al. 2001). It is well known that the obliquity cycle of the earth with a major periodicity of $41 \mathrm{k}$ years modulates the Northern and Southern Hemispheric warming. This might shift the ITZC meridionally, thereby affecting the strength of the annual cycle in the Tropics. Due to the nonlinear rectification effect mentioned above these changes might feed back onto the tropical climate mean state, generating different SST patterns. These speculations will be investigated further in subsequent studies by comparing model simulations with paleo data.

Another exciting perspective created by our nonlinear theory is that of regime predictability. Return maps of large amplitude events can be constructed, offering the possibility to predict the next large El Niño or La Niña event of given amplitude. As can be seen from Fig. 1, the dynamics of ENSO's envelope can be divided crudely into a low and a high variance regime. Hence, the prediction of the next large El Niño event is similar to predicting the next high amplitude ENSO regime. One crucial finding here is that, due to the structure of return time maps (not shown), namely the existence of "preferred" and "forbidden" return times, information on long-term El Niño bursts can be made based on the topological structure of the attractor. Our approach offers the possibility to explore this kind of nonlinear predictability.

We are fully aware that our low-order ENSO model cannot capture all physical processes involved in tropical climate dynamics. The El Niño bursting simulated by our simple ENSO model is stronger than the one seen in observations (Torrence and Webster 1999). Nevertheless, the use of such a simple ENSO model is justified as it helps to gain more insight into generic nonlinear processes and to generate new ideas.

It should be noted here that the inclusion of an external wind stress, which is unrelated to the east-west temperature gradient, leads to a symmetry breaking of the radiative-convective equilibrium state (Jin 1996). This will also modify the interaction between the RC 
state and the cold tongue climate state, being at the core of our El Niño bursting mechanism. Experiments have shown that a realistic external wind stress forcing (due to the Hadley circulation) affects the amplitude modulations for small values of the advection strength, but has relatively little effect on the strong advection case. Overall the effect of this external forcing is to shrink the area of amplitude modulations in parameter space shown in Fig. 6. A more detailed analysis of this symmetry breaking effect on the amplitude and frequency of ENSO will be described in a subsequent study. So far, it is not certain, how important this effect is in suppressing ENSO bursting in reality or in more complex climate models.

Our future research will focus on establishing this mechanism for El Niño bursting using models of intermediate complexity such as the Zebiak and Cane (1987) model. Furthermore, we will study the nonlinear predictability of decadal ENSO amplitude modulations more in detail.

Acknowledgments. We thank Diane Henderson for her help editing this manuscript. We are grateful to Nancy Hulbirt and Annegreth Schurbohm for their graphical assistance. Part of the work was done at the International Pacific Research Center (IPRC). The IPRC is supported in part by the Frontier Research System for Global Change. A. Timmermann's work is now funded by the German Science Foundation (DFG). F.-F. Jin is supported by NOAA and NSF grants. The reviewers helped to improve this manuscript significantly. We appreciate their contributions. In addition we are very grateful to Dr. Soon-II An for estimating the $\epsilon$ value from SODA data.

\section{APPENDIX}

\section{Homoclinic and Heteroclinic Orbits}

A very important step toward understanding the generation of nonlinear amplitude modulations in dynamical systems is the identification of saddle points and saddle foci corresponding to the underlying dynamical equations. A saddle point in two dimensions is a fixed point that is characterized by one positive and one negative eigenvalue. A saddle focus in three-dimensions is a fixed point of the system that is characterized by a pair of complex conjugated eigenvalues with negative (positive) real part and a positive (negative) real eigenvalue.

1) Two-dimensional homoclinic and heteroclinic connections: A homoclinic orbit connects a saddle point with itself.

A heteroclinic orbit in two dimensions links two saddle points with each other as shown in Fig. 13b. In both cases the period is infinity.

2) Three-dimensional homoclinic and heteroclinic connections: In three-dimensions two types of homo- clinic orbits exist: one characterized by a saddle point connection (Fig. 13c) and another characterized by saddle focus self-connection (see Fig. 13d). In addition, a saddle focus can be connected with a saddle point to form a heteroclinic connection (Fig. 13e). This is exactly the case for our low-order ENSO system that exhibits amplitude modulated behavior when the saddle focus is interacting with a saddle point representing the unstable radiative-convective equilibrium.

Each of the situations displayed in Fig. 13 can be decomposed into a normal time and a time-inversed case. In addition, the ratio of the eigenvalues of the connected saddle points is a further characteristic of the connection. It is beyond the scope of this paper to give a detailed description of all these type of orbits. The interested reader is referred to the book of Wiggins (1988). It is important to note that, in many cases which involve saddle focus connections, chaos is a generic feature in the parameter vicinity of the homoclinic/heteroclinic orbits. In addition, we have seen that connections involving a saddle focus naturally generate amplitude modulations as a result of the complex eigenvalue and the reinjection into the saddle focus.

\section{REFERENCES}

An, S.-I., F.-F. Jin, and I.-S. Kang, 1999: The role of zonal advection feedback in phase transition and growth of ENSO in the CaneZebiak model. J. Meteor. Soc. Japan, 77, 1151-1160.

Blanke, B., J. D. Neelin, and D. Gutzler, 1997: Estimating the effect of stochastic wind stress forcing on ENSO irregularity. J. Climate, 10, 1473-1487.

Cane, M. A., 1979: The response of an equatorial ocean to simple wind stress patterns. Part 1: Model formulation and analytical results. J. Mar. Sci., 37, 233-252.

Cane, M. E., and E. S. Sarachik, 1981: The response of a linear baroclinic equatorial ocean to periodic forcing. J. Mar. Res., 39, 651-693.

Carton, J. A., G. Chepurin, and X. Cao, 2000: A simple ocean data assimilation analysis of the global upper ocean 1950-1995. Part II: Results. J. Phys. Oceanogr., 30, 311-326.

Chang, P., J. Link, L. Hong, and M. Flügel, 1996: Chaotic dynamics versus stochastic processes in El Niño-Southern Oscillation in coupled ocean-atmosphere models. Physica D, 98, 301-320.

Doedel, E. J., 1981: AUTO, a program for the automatic bifurcation analysis of autonomous systems. Congr. Numer., 30, 265-384.

_ H. B. Keller, and J. P. Kernevez, 1991: Numerical analysis and control of bifurcation problems. (I) Bifurcation in finite dimensions. Int. J. Bifurcation Chaos, 1, 493-520.

Douglass, D. H., D. R. Abrams, D. M. Baranson, and B. D. Clader, cited 2002: On the Nature of the El Niño/La Niña events. Los Alamos Preprint physics/0203016, 22 pp. [Available online at http://arxiv.org/abs/physics/0203016.]

Eckert, C., and M. Latif, 1997: Predictability of a stochastically forced hybrid coupled model of El Niño. J. Climate, 10, 1488-1504.

Glenndinning, P., and C. Sparrow, 1984: Local and global behavior near homoclinic orbits. J. Stat. Phys., 35, 645-697.

Graham, N. E., 1994: Decadal-scale climate variability in the 1970 s and 1980s: Observations and model results. Climate Dyn., 10, 135-159.

Gu, D.-F., and S. G. H. Philander, 1995: Secular changes of annual and interannual variability in the Tropics during the past century. J. Climate, 8, 864-876. 
- , and — 1997: Interdecadal climate fluctuations that depend on exchanges between the tropics and extratropics. Science, 275, 805-807.

Jin, F.-F., 1996: Tropical ocean-atmosphere interaction, the Pacific cold tongue, and the El Niño-Southern Oscillation. Science, 274, $76-78$.

- 1997: An equatorial ocean recharge paradigm for ENSO. Part I: Conceptual model. J. Atmos. Sci., 54, 811-829.

_ 1998: A simple model for the Pacific cold tongue and ENSO. J. Atmos. Sci., 55, 2458-2469.

_ within the equatorial ocean recharge oscillator model for ENSO. Geophys. Res. Lett., 26, 2989-2992.

_ case: Annual subharmonic steps to chaos. Science, 264, 70-72.

Kirtman, B. P., and P. S. Schopf, 1998: Decadal variability in ENSO predictability and prediction. J. Climate, 11, 2804-2822.

Kleeman, R., J. P. McCreary Jr., and B. A. Klinger, 1999: A mechanism for generating ENSO decadal variability. Geophys. Res. Lett., 26, 1743-1746.

Liu, X., and R. H. Zhang, 1999: Propagation and mechanism of decadal upper ocean variability in the North Pacific. Geophys. Res. Lett., 26, 739-742.

Moore, A. M., and R. Kleeman, 1999: Stochastic forcing of ENSO by the intraseasonal oscillation. J. Climate, 12, 1199-1220.

Münnich, M., M. A. Cane, and S. E. Zebiak, 1991: A study of selfexcited oscillations of the tropical ocean-atmosphere system. Part II: Nonlinear cases. J. Atmos. Sci., 48, 1238-1248.

Neelin, J. D., D. S. Battisti, A. C. Hirst, F. F. Jin, Y. Wakata, T. Yamagata, and S. E. Zebiak, 1998: ENSO theory. J. Geophys. Res., 103 (C7), 14 261-14 290.

Pierce, D. W., T. P. Barnett, and M. Latif, 1999: Connections between the Pacific Ocean Tropics and midlatitudes on decadal timescales. J. Climate, 13, 1173-1194.

Schneider, N., 2000: A decadal spiciness mode in the Tropics. Geophys. Res. Lett., 27, 257-260.

Shil'nikov, L. P., 1965: A case of the existence of a denumerable set of periodic motions. Sov. Math. Dokl., 6, 163-166.

- 1970: A contribution to the problem of the structure of an extended neighborhood of a rough equilibrium state of saddlefocus type. Math. USSR Sbornik, 10, 91-102.

Sun, D. Z., 2000: Global climate change and ENSO: A theoretical framework. El Niño: Historical and Paleoclimatic Aspects of the Southern Oscillation, Multiscale Variability and Global and Regional Impacts. H. F. Diaz and V. Markgraf, Eds., Cambridge University Press, 443-463.

Timmermann, A., 2002: Decadal ENSO amplitude modulations: A nonlinear mechanism. Global Planet. Change, in press.

_ , and F.-F. Jin, 2002: A nonlinear mechanism for decadal El Niño amplitude changes. Geophys. Res. Lett., 29, 1003, doi: 10.1029/ 2001GL013369.

_ M. Latif, A. Bacher, J. Oberhuber, and E. Roeckner, 1999: Increased El Niño frequency in a climate model forced by future greenhouse warming. Nature, 398, 694-696.

-, H. U. Voss, and R. Pasmanter, 2001: Empirical dynamical system modeling of ENSO using nonlinear inverse techniques. J. Phys. Oceanogr., 31, 1579-1598.

Torrence, C., and P. J. Webster, 1999: Interdecadal changes in the ENSO-monsoon system. J. Climate, 12, 2679-2690.

Tudhope, A., and Coauthors, 2001: Variability in the El Niño-Southern Oscillation through a glacial-interglacial cycle. Science, 291, $1511-1517$

Tziperman, E., L. Stone, M. A. Cane, and H. Jarosh, 1994: El Niño chaos: Overlapping of resonances between the seasonal cycle and the Pacific Ocean-atmosphere oscillator. Science, 264, $72-74$.

Wang, B., A. Barcilon, and Z. Fang, 1999: Stochastic dynamics of El Niño-Southern Oscillation. J. Atmos. Sci., 56, 5-23.

Weaver, A. J., 1999: Extratropical subduction and decadal modulation of El Niño. Geophys. Res. Lett., 26, 743-746.

Wiggins, S., 1988: Global Bifurcations and Chaos: Analytical Methods. Applied Mathematical Sciences Series, Vol. 73, SpringerVerlag, $494 \mathrm{pp}$.

Zebiak, S. E., and M. A. Cane, 1987: A model El Niño-Southern Oscillation. Mon. Wea. Rev., 115, 2262-2278.

Zhang, R. H., L. M. Rothstein, and A. J. Busalacchi, 1998: Origin of upper-ocean warming and El Niño change on decadal scales in the tropical Pacific Ocean. Nature, 391, 879-883.

Zhang, Y., J. M. Wallace, and D. S. Battisti, 1997: ENSO-like interdecadal variability: 1900-93. J. Climate, 10, 1004-1020. 